Commun. Korean Math. Soc. 24 (2009), No. 2, pp. 161-169

DOI 10.4134/CKMS.2009.24.2.161

\title{
A GENERALIZED IDEAL BASED-ZERO DIVISOR GRAPHS OF NEAR-RINGS
}

\author{
Patchirajulu Dheena and Balasubramanian Elavarasan
}

\begin{abstract}
In this paper, we introduce the generalized ideal-based zerodivisor graph structure of near-ring $N$, denoted by $\widehat{\Gamma_{I}(N)}$. It is shown that if $I$ is a completely reflexive ideal of $N$, then every two vertices in $\widehat{\Gamma_{I}(N)}$ are connected by a path of length at most 3 , and if $\widehat{\Gamma_{I}(N)}$ contains a cycle, then the core $K$ of $\widehat{\Gamma_{I}(N)}$ is a union of triangles and rectangles. We have shown that if $\widehat{\Gamma_{I}(N)}$ is a bipartite graph for a completely semiprime ideal $I$ of $N$, then $N$ has two prime ideals whose intersection is $I$.
\end{abstract}

\section{Preliminaries}

Throughout this paper, $N$ denotes a zero-symmetric near-ring not necessarily with identity unless otherwise stated. For any vertices $x, y$ in a graph $G$, if $x$ and $y$ are adjacent, we denote it as $x \approx y$. In [3], Beck introduced the concept of a zero-divisor graph of a commutative ring with identity, but this work was mostly concerned with coloring of rings. In [2], Anderson and Livingston associate to a commutative ring with identity a (simple) graph $\Gamma(R)$, whose vertex set is $Z(R)^{*}=Z(R) \backslash\{0\}$, the set of nonzero-divisor of $R$, in which two distinct $x, y \in Z(R)^{*}$ are joined by an edge if and only if $x y=0$. They investigated the interplay between the ring-theoretic properties of $R$ and the graph-theoretics properties of $\Gamma(R)$. The zero-divisor graph has also been introduced and studied for semigroups by DeMeyer et al. in [7].

In [11], Redmond has generalized the notion of the zero-divisor graph. For a given ideal $I$ of a commutative ring $R$, he defined an undirected graph $\Gamma_{I}(R)$ with vertices $\{x \in R \backslash I: x y \in I$ for some $y \in R \backslash I\}$, where distinct vertices $x$ and $y$ are adjacent if and only if $x y \in I$. In [8], Dheena and Elavarasan extended this graph structure to near-rings. Following [8], let $I$ be a completely reflexive ideal (i.e., $a b \in I$ implies $b a \in I$ for $a, b \in N$ ) of $N$. Then the ideal-based zero-divisor graph, denoted by $\Gamma_{I}(N)$, is the graph whose vertices are the set

Received February 12, 2008; Revised August 6, 2008.

2000 Mathematics Subject Classification. 16Y30, $13 \mathrm{~A} 15$.

Key words and phrases. ideal-based zero-divisor graph, diameter, near-ring, ideal and cycle.

(C)2009 The Korean Mathematical Society 
$\{x \in N \backslash I: x y \in I$ for some $y \in N \backslash I\}$ with distinct vertices $x$ and $y$ are adjacent if and only if $x y \in I$.

In this paper, we define a generalized ideal-based zero-divisor graph structure of the near-ring $N$. Let $N$ be a near-ring and $I$ be a completely reflexive ideal of $N$. We define an undirected graph $\widehat{\Gamma_{I}(N)}$ with vertices $\{x \in N \backslash I$ : there exists $y \in N \backslash I$ such that $x_{1} y_{1} \in I$ for some $x_{1} \in\langle x\rangle \backslash I$ and $\left.y_{1} \in\langle y\rangle \backslash I\right\}$, where distinct vertices $x$ and $y$ are adjacent if and only if $x_{1} y_{1} \in I$ for some $x_{1} \in\langle x\rangle \backslash I$ and $y_{1} \in\langle y\rangle \backslash I$, where $\langle x\rangle$ denotes the ideal of $N$ generated by $x$.

Clearly $\Gamma_{I}(N)$ is a induced subgraph of $\widehat{\Gamma_{I}(N)}$, and if $I=\{0\}$, then $\widehat{\Gamma_{I}(N)}$ will be denoted simply by $\widehat{\Gamma(N)}$. Also, $\widehat{\Gamma_{I}(N)}=\phi$ if and only if $I$ is a prime ideal of $N$. That is, $V\left(\widehat{\Gamma_{I}(N)}\right)=\phi$ if and only if $V(\widehat{\Gamma(N / I)})=\phi$. Observe that $\left|V\left(\Gamma_{I}(N)\right)\right|=0$ if and only if $\left|V\left(\widehat{\Gamma_{I}(N)}\right)\right|=0$. Also $\left|V\left(\Gamma_{I}(N)\right)\right| \leq\left|V\left(\widehat{\Gamma_{I}(N)}\right)\right|$.

Example 1.1. Below are the generalized zero-divisor graphs for several nearrings. Note that these examples show that the graph structures $\Gamma_{I}(N)$ and $\widehat{\Gamma_{I}(N)}$ are not isomorphic and non-isomorphic near-rings may have the isomorphic generalized zero-divisor graph.

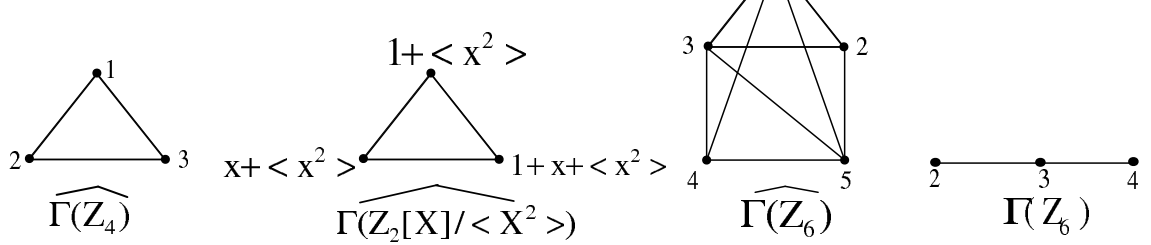

Given a graph $G$, for distinct vertices $x$ and $y$ of $G$, let $d(x, y)$ be the length of the shortest path from $x$ to $y$. The diameter of a connected graph is the supremum of the distances between vertices. The core $K$ of $G$ is the union of all cycles of $G$. From [8], for any subset $S$ and ideal $I$ of $N$, we define $I_{S}=\{n \in N: n S \subseteq I\}$. If $S=\{a\}$, then we denote $I_{\{a\}}$ by $I_{a}$. In this paper the notations of graph theory are from [5], the notations of near-ring are from [10].

\section{Main results}

Theorem 2.1. Let $I$ be a completely reflexive ideal of $N$. Then $\Gamma_{I}(N)$ is a connected graph and $\operatorname{diam}\left(\Gamma_{I}(N)\right) \leq 3$.

Proof. The sketch of this proof follows in the similar manner to the proof of Theorem 2.4 of [11].

Lemma 2.2. Let $I$ be a completely reflexive ideal of $N$. For any $x, y \in \widehat{\Gamma_{I}(N)}$, if $x \approx y$ is an edge in $\widehat{\Gamma_{I}(N)}$, then for each $n \in N \backslash I$, either $n \approx y$ or $x \approx y^{\prime}$ is an edge in $\widehat{\Gamma_{I}(N)}$ for some $y^{\prime} \in\langle y\rangle \backslash I$. 
Proof. Let $x, y \in N \backslash I$ with $x \approx y$ be an edge in $\widehat{\Gamma_{I}(N)}$ and suppose that $n \approx y$ is not an edge in $\widehat{\Gamma_{I}(N)}$ for some $n \in N \backslash I$. Then $x_{1} y_{1} \in I$ for some $x_{1} \in\langle x\rangle \backslash I ; y_{1} \in\langle y\rangle \backslash I$ and $n y_{1} \notin I$. But $\left(n y_{1}\right) x_{1} \in I$. So $x \approx y^{\prime}$ is an edge in $\widehat{\Gamma_{I}(N)}$ for some $y^{\prime} \in\langle y\rangle \backslash I$.

Theorem 2.3. Let I be a completely reflexive ideal of $N$. Then $\widehat{\Gamma_{I}(N)}$ is connected graph with $\operatorname{diam}\left(\widehat{\Gamma_{I}(N)}\right) \leq 3$.

Proof. Let $x, y \in \widehat{\Gamma_{I}(N)}$. If $x_{1} y_{1} \in I$ for some $x_{1} \in\langle x\rangle \backslash I$ and $y_{1} \in\langle y\rangle \backslash I$, then $d(x, y)=1$. Let us assume that $x_{1} y_{1} \notin I$ for all $x_{1} \in\langle x\rangle \backslash I$ and for all $y_{1} \in$ $\langle y\rangle \backslash I$. Then $x_{1}^{2} \notin I$ and $y_{1}^{2} \notin I$ for all $x_{1} \in\langle x\rangle \backslash I$ and for all $y_{1} \in\langle y\rangle \backslash I$. Since $x, y \in \widehat{\Gamma_{I}(N)}$, there exist $x_{2} \in\langle x\rangle \backslash I ; y_{2} \in\langle y\rangle \backslash I$ and $a_{1}, b_{1} \in N \backslash\left(I \cup\left\{x_{2}, y_{2}\right\}\right)$ such that $a_{1} x_{2} \in I$ and $b_{1} y_{2} \in I$.

If $a_{1}=b_{1}$, then $x \approx a_{1} \approx y$ is a path of length 2 . So assume that $a_{1} \neq b_{1}$.

If $a_{1} b_{1} \in I$, then $x \approx a_{1} \approx b_{1} \approx y$ is a path of length 3. Otherwise $a_{1} b_{1} \notin I$. Then $\left\langle a_{1}\right\rangle \cap\left\langle b_{1}\right\rangle \nsubseteq I$. Now for every $d \in\left\langle a_{1}\right\rangle \cap\left\langle b_{1}\right\rangle \backslash\left(I \cup\left\{x_{2}, y_{2}\right\}\right)$, we have $d x_{2} \in\langle d\rangle\left\langle x_{2}\right\rangle \subseteq\left\langle a_{1}\right\rangle\left\langle x_{2}\right\rangle \subseteq I$ and $d y_{2} \in\left\langle b_{1}\right\rangle\left\langle y_{2}\right\rangle \subseteq I$. Thus $x \approx d \approx y$ is a path of length 2 and hence $\Gamma_{I}(N)$ is connected and $\operatorname{diam}\left(\widehat{\Gamma_{I}(N)} \leq 3\right.$.

Theorem 2.4. Let $I$ be a completely reflexive ideal of $N$ and if $a \approx x \approx b$ is a path in $\widehat{\Gamma_{I}(N)}$, then either $I \cup\left\{x_{1}\right\}$ is an ideal of $N$ for some $x_{1} \in\langle x\rangle \backslash I$ or $a \approx x \approx b$ is contained in a cycle of length $\leq 4$.

Proof. Let $a \approx x \approx b$ be a path in $\widehat{\Gamma_{I}(N)}$. Then there exist $x_{1}, x_{2} \in\langle x\rangle \backslash I ; a_{1} \in$ $\langle a\rangle \backslash I$ and $b_{1} \in\langle b\rangle \backslash I$ such that $a_{1} x_{1} \in I$ and $b_{1} x_{2} \in I$. If $a^{\prime} b^{\prime} \in I$ for some $a^{\prime} \in\langle a\rangle \backslash I$; for some $\langle b\rangle \backslash I$, then $a \approx x \approx b \approx a$ is contained in a cycle of length $\leq 4$. So let us assume that $a_{1} b_{1} \notin I$ for all $a_{1} \in\langle a\rangle \backslash I$ and $b_{1} \in\langle b\rangle \backslash I$.

Case (i) Let $x_{1}=x_{2}$. Then either $I_{a_{1}} \cap I_{b_{1}}=I \cup\left\{x_{1}\right\}$ or there exists $c \in I_{a_{1}} \cap I_{b_{1}}$ such that $c \notin I \cup\left\{x_{1}\right\}$. Then $c a_{1}, c b_{1} \in I$. In the first case, $I \cup\left\{x_{1}\right\}$ is an ideal. In the second case $a \approx x \approx b \approx c \approx a$ is contained in a cycle of length $\leq 4$.

Case (ii) Let $x_{1} \neq x_{2}$. Then clearly $\left\langle a_{1}\right\rangle \cap\left\langle b_{1}\right\rangle \nsubseteq I$. Then for each $z \in$ $\left\langle a_{1}\right\rangle \cap\left\langle b_{1}\right\rangle \backslash I$, we have $z x_{1} \in\left\langle a_{1}\right\rangle\left\langle x_{1}\right\rangle \subseteq I$ and $z x_{2} \in I$. Clearly either $x_{1} \neq x$ or $x_{2} \neq x$. Say $x_{1} \neq x$. Then we have a path $a \approx x_{1} \approx b$ and hence $a \approx x \approx$ $b \approx x_{1} \approx a$ is contained in a cycle of length $\leq 4$.

In Theorem 2.4, the bound for the length of the cycle is sharp as the following example shows.

Example 2.5. Let $N=\left(\begin{array}{cc}F & F \\ 0 & F\end{array}\right)$, where $F=\{0,1\}$ is the field under addition and multiplication modulo 2. Then it's prime radical $P=\left\{\left(\begin{array}{ll}0 & 0 \\ 0 & 0\end{array}\right),\left(\begin{array}{ll}0 & 1 \\ 0 & 0\end{array}\right)\right\}$ is a completely reflexive ideal of the near-ring $N$ and its generalized zero-divisor graph $\widehat{\Gamma_{P}(N)}$ is: 


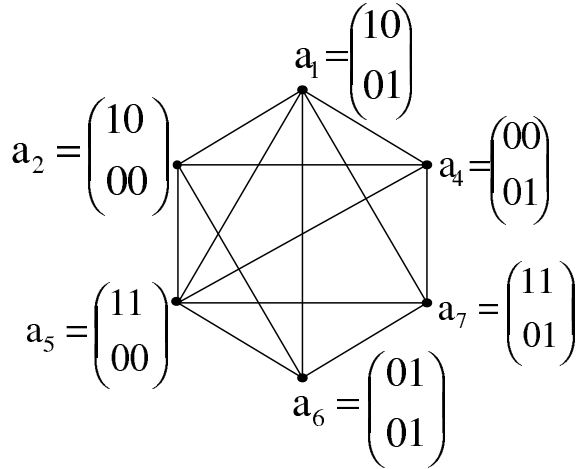

It is easy to verify that $P \cup\{a\}$ is not an ideal of $N$ for any $a \in\left\langle a_{2}\right\rangle \backslash P$ and $a_{4} \approx a_{2} \approx a_{6}$ is not contained in cycle of length 3 .

Corollary 2.6. Let I be a completely reflexive ideal of $N$ and $\left|V\left(\Gamma_{I}(N)\right)\right|>2$. If $I \cup\{x\}$ is not an ideal of $N$ for any $x \in N \backslash I$, then every edge in $\widehat{\Gamma_{I}(N)}$ is contained in a cycle of length $\leq 4$, and therefore $\widehat{\Gamma_{I}(N)}$ is a union of triangles and squares.

Lemma 2.7. Let I be a completely reflexive ideal of $N$. Then, $\widehat{\Gamma_{I}(N)}$ can be neither a pentagon nor a hexagon.

Proof. Suppose that $\widehat{\Gamma_{I}(N)}$ is $a \approx b \approx c \approx d \approx e \approx a$, a pentagon. Then by Theorem 2.4, for one of the vertices (say b), $I \cup\left\{b_{1}\right\}$ is an ideal of $N$ for some $b_{1} \in\langle b\rangle \backslash I$. Then in the pentagon, there exist $d_{1} \in\langle d\rangle \backslash I$ and $e_{1} \in\langle e\rangle \backslash I$ such that $d_{1} e_{1} \in I$. Since $I \cup\left\{b_{1}\right\}$ is ideal, $b_{1} d_{1}=b_{1}=b_{1} e_{1}$. But $b_{1}\left(d_{1} e_{1}\right) \in I$, then $b_{1} \in I$, a contradiction. The proof for the hexagon is the same.

Theorem 2.8. Let I be a completely reflexive ideal of $N$. Then the following hold:

(i) If $N$ has identity, then $\widehat{\Gamma_{I}(N)}$ has no cut-vertices.

(ii) If $N$ has no identity and if I is non-zero ideal of $N$, then $\widehat{\Gamma_{I}(N)}$ has no cut-vertices.

Proof. Suppose that the vertex $x$ of $\widehat{\Gamma_{I}(N)}$ is a cut vertex. Let $u \approx x \approx w$ be a path in $\widehat{\Gamma_{I}(N)}$. Since $x$ is a cut-vertex, $x$ lies in every path from $u$ to $w$.

(i) Assume that $N$ is a near-ring with identity.

For any $u, v \in \widehat{\Gamma_{I}(N)}$, there exist a path $u \approx 1 \approx w$ which shows $x(\neq 1)$ in $\widehat{\Gamma_{I}(N)}$ is not a cut vertex. Suppose $x=1$. Then there exist $u_{1} \in\langle u\rangle \backslash I ; w_{1} \in$ $\langle w\rangle \backslash I$ and $t_{1}, t_{2} \in N \backslash I$ such that $u_{1} t_{1}, w_{1} t_{2} \in I$ which implies $u_{1}, w_{1} \in \Gamma_{I}(N)$. Since $\Gamma_{I}(N)$ is connected, there exist $n, n_{1} \in N \backslash(I \cup\{x\})$ such that $u_{1} \approx n \approx w_{1}$ or $u_{1} \approx n \approx n_{1} \approx w_{1}$ is a path in $\Gamma_{I}(N)$ which implies $u \approx n \approx w \approx 1 \approx u$ or $u \approx n \approx n_{1} \approx w \approx 1 \approx u$ is a cycle in $\widehat{\Gamma_{I}(N)}$, contradicting $x=1$ is a cut-vertex. 
(ii) Let $N$ be a near-ring without identity and $I$ be a non-zero ideal of $N$.

Since $u \approx x \approx w$ is a path from $u$ to $w$, then there exist $u_{1} \in\langle u\rangle \backslash I ; w_{1} \in$ $\langle w\rangle \backslash I$ and $x_{1}, x_{2} \in\langle x\rangle \backslash I$ such that $u_{1} x_{1} \in I$ and $w_{1} x_{2} \in I$.

Case (i) $x_{1}=x_{2}$

If $u_{1}+I=x_{1}+I$, then $u_{1} w_{1} \in I$ which implies $u$ is adjacent to $w$. Similarly, if $x_{2}+I=w_{1}+I, u$ is adjacent to $w$. So assume that $u_{1}+I \neq x_{1}+I$ and $x_{2}+I \neq w_{1}+I$. Let $0 \neq i \in I$. Then $u_{1} x_{1} \in I$ and $w_{1} x_{2} \in I$ which imply that $u_{1}\left(x_{1}+i\right), w_{1}\left(x_{1}+i\right) \in I$. If $x=x_{1}+i$, then $x \neq x_{1}$ which implies $u \approx x_{1} \approx w$ is a path in $\widehat{\Gamma_{I}(N)}$. Otherwise $u \approx\left(x_{1}+i\right) \approx w$ is a path in $\widehat{\Gamma_{I}(R)}$. Thus there exist a path from $u$ to $w$ not passing through $x$, a contradiction.

Case (ii) Either $x_{1}$ or $x_{2}$ equal to $x$.

Without loss of generality, let us assume that $x_{1}=x$ and $x_{2} \neq x$. Then $u_{1} x \in I$ and $x_{2} w_{1} \in I$ which implies $u_{1} x_{2} \in I$ and $x_{2} w_{1} \in I$, and so we have a path $u \approx x_{2} \approx w$, a contradiction.

Case (iii) Neither $x_{1}$ nor $x_{2}$ equal to $x$.

If $x_{1} x_{2} \in I$, then we have a path $u \approx x_{1} \approx x_{2} \approx w$, a contradiction. Otherwise $x_{1} x_{2} \notin I$.

If $x_{1} x_{2}=x$, then $u_{1} x \in I$ and $w_{1} x \in I$. By sub case (i), we have a contradiction.

So assume that $x_{1} x_{2} \neq x$, then we have a path $u \approx x_{1} x_{2} \approx w$, a contradiction.

Thus $x$ can not be a cut-vertex.

From Theorem 2.8, we have the following question. If $N$ is a near-ring without identity and $\{0\}$ is a completely reflexive ideal of $N$, then whether $\widehat{\Gamma(N)}$ has a cut-vertex.

Theorem 2.9. Let I be a completely reflexive ideal of $N$. If $\widehat{\Gamma_{I}(N)}$ contains a cycle, then the core $K$ of $\widehat{\Gamma_{I}(N)}$ is a union of triangles and rectangles. Moreover, any vertex in $\widehat{\Gamma_{I}(N)}$ is either a vertex of the core $K$ of $\widehat{\Gamma_{I}(N)}$ or else is an end vertex of $\widehat{\Gamma_{I}(N)}$.

Proof. Let $a \in K$ and assume that $a$ is not in any square or rectangle in $\widehat{\Gamma_{I}(N)}$. Then $a$ is part of a cycle $a \approx b \approx c \approx d \approx \cdots \approx a$ which implies $c_{1} d_{1} \in I$ for

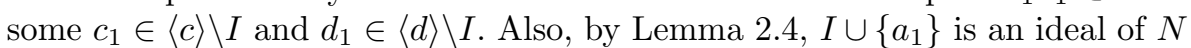
for some $a_{1} \in\langle a\rangle \backslash I$. Then $d_{1} a_{1}=a_{1}=c_{1} a_{1}$ and $a_{1}\left(d_{1} c_{1}\right) \in I$ which implies $a_{1} \in I$, a contradiction.

For the "moreover"statement, we can assume $\left|\widehat{\Gamma_{I}(N)}\right| \geq 3$. If $x$ is a vertex in $\widehat{\Gamma_{I}(N)}$, then one of the following is true:

1. $x$ is in the core;

2. $x$ is an end vertex of $\widehat{\Gamma_{I}(N)}$;

3. $a \approx x \approx b$ is a path in $\widehat{\Gamma_{I}(N)}$ where $a$ is an end vertex and $b \in K$; 
4. $a \approx x \approx y \approx b$ or $a \approx y \approx x \approx b$ is a path in $\widehat{\Gamma_{I}(N)}$, where $a$ is an end vertex and $b \in K$.

In the first two cases, we are done. Let us assume that $a \approx x \approx b$ is a path with $b \in K$. Then by Lemma 2.4, $I \cup\left\{x_{1}\right\}$ is an ideal of $N$ for some $x_{1} \in\langle x\rangle \backslash I$ and $x \approx b \approx c \approx d \approx b$ or $x \approx b \approx c \approx d \approx e \approx b$ is a path in $\widehat{\Gamma_{I}(N)}$ which implies $c_{1} d_{1} \in I$ for some $c_{1} \in\langle c\rangle \backslash I$ and $d_{1} \in\langle d\rangle \backslash I$. Since $x \notin K$, we have $x_{1} c_{1}=x_{1}$ and so $x$ is a vertex in the cycle $x \approx b \approx c \approx d \approx x$, a contradiction.

Although the proof of case 4 is just a slight modification of that for Theorem 2.4 given in [6], we include a sketch of the proof to illustrate the style.

Without loss of generality, assume $a \approx x \approx y \approx b$ is a path in $\widehat{\Gamma_{I}(N)}$. Since $b \in K$, there is some $c \in K$ such that $c \neq b$ and $b \approx c$ is part of a cycle. Then $a \approx x \approx y \approx b \approx c$ is a path in $\widehat{\Gamma_{I}(N)}$. But the distance from $a$ to $c$ is four, a contradiction unless $y \approx c$ or $x \approx c$ is an edge. However, if $y \approx c$ is an edge, then $y \in K$. By case $3, x$ is also in the core. If instead, $x \approx c$ is an edge, then $x \approx y \approx b \approx c \approx x$ is a cycle. Thus $x, y \in K$.

Hence it must be the case that any vertex $x$ of $\widehat{\Gamma_{I}(N)}$ is either an end or in the core.

Corollary 2.10. Let I be a completely reflexive ideal of $N$. If $N$ has identity with $\left|V\left(\Gamma_{I}(N)\right)\right| \geq 2$ or if $I$ is non-zero ideal of $N$ with $\left|V\left(\Gamma_{I}(N)\right)\right|>2$, then $\widehat{\Gamma_{I}(N)}=K$, where $K$ is the core of $\widehat{\Gamma_{I}(N)}$.

Corollary 2.11. Let I be a completely reflexive ideal of $N$ with $\left|V\left(\Gamma_{I}(N)\right)\right|>$ 2. If $I \cup\{x\}$ is not an ideal of $N$, then every pair of vertices in $\widehat{\Gamma_{I}(N)}$ is contained in a cycle of length $\leq 6$.

Proof. Let $a, b$ be vertices of $\widehat{\Gamma_{I}(N)}$. If $a \approx b$ is an edge in $\widehat{\Gamma_{I}(N)}$, then $a \approx b$ is the edge of a triangle or rectangle by Corollary 2.6. If $a \approx x \approx y$ is a path in $\widehat{\Gamma_{I}(N)}$, then $a \approx x \approx b$ is contained in a cycle of length $\leq 4$ by Theorem 2.4. If $a \approx x \approx y \approx b$ is a path in $\widehat{\Gamma_{I}(N)}$, then by Lemma 2.4 we can find cycles $a \approx x \approx y \approx c \approx a$ and $b \approx y \approx x \approx d \approx b$, where $c \neq x$ and $d \neq y$. This gives a cycle $a \approx x \approx d \approx b \approx y \approx c \approx a$ of length $\leq 6$.

Recall that a bipartite graph is one whose vertex set can be partitioned into two subsets so that no edge has both ends in any one subset. We now obtain the properties of a near-ring implied by its generalized ideal-based zero-divisor graph.

Theorem 2.12. Let $I$ be a completely reflexive ideal of $N$ and $I$ is completely semiprime. If $\widehat{\Gamma_{I}(N)}$ is a bipartite graph, then there exist prime ideals $P_{1}$ and $P_{2}$ of $N$ such that $I=P_{1} \cap P_{2}$.

Proof. Let $A, B$ be the partition of the graph $\widehat{\Gamma_{I}(N)}$. Let $V_{1}=\{x \in A$ is a vertex in $\widehat{\Gamma_{I}(N)}$ such that $x y \in I$ for some $\left.y \in B\right\}$ and $V_{2}=\{y \in B$ is a 
vertex in $\widehat{\Gamma_{I}(N)}$ such that $x y \in I$ for some $\left.y \in A\right\}$. Observe that $V_{1}$ and $V_{2}$ are non-empty and also $V_{1} \cap I=V_{2} \cap I=\phi$. Let $P_{1}=V_{1} \cup I$ and $P_{2}=V_{2} \cup I$. Then $I=P_{1} \cap P_{2}$. Let us show that $P_{1}$ is an ideal of $N$. Let $x_{1}, x_{2} \in P_{1}$.

Case (i): If $x_{1}$ and $x_{2}$ are in $I$, then $x_{1}-x_{2} \in I \subseteq P_{1}$.

Case (ii): Let $x_{1}, x_{2} \in V_{1}$. If $x_{1}-x_{2} \in I$, then $x_{1}-x_{2} \in P_{1}$. Hence let us assume that $x_{1}-x_{2} \notin I$. Now there exist $y_{1}, y_{2} \in V_{2}$ such that $x_{1} y_{1} \in I$ and $x_{1} y_{2} \in I$. Hence $x_{1} y_{1} y_{2} \in I$ and $x_{2} y_{1} y_{2} \in I$. If $y_{1} y_{2} \in I$, then $y_{1} \approx y_{2}$ contradicts to the fact that no two vertices in $B$ are adjacent. Hence $y_{1} y_{2} \notin I$. Clearly $x_{1}-x_{2} \notin V_{2}$ and $x_{1}-x_{2} \neq y_{1} y_{2}$. Indeed, if $x_{1}-x_{2} \in V_{2}$ or $x_{1}-x_{2}=y_{1} y_{2}$, then $\left(x_{1}-x_{2}\right) y_{1} y_{2} \in I$ or $\left(y_{1} y_{2}\right)^{2} \in I$, a contradiction since $y_{1} y_{2} \in V_{2}$. Now $\left(x_{1}-x_{2}\right) y_{1} y_{2} \in I$ with $x_{1}-x_{2} \notin I, y_{1} y_{2} \notin I$. Thus $x_{1}-x_{2} \in A$ and $y_{1} y_{2} \in B$ and hence $x_{1}-x_{2} \in V_{1} \subseteq P_{1}$.

Case (iii): Suppose $x_{1} \in V_{1}$ and $x_{2} \in I$. Clearly $x_{1}-x_{2} \notin I$. Since $x_{1} \in V_{1}$, there exists $y_{1} \in V_{2}$ such that $x_{1} y_{1} \in I$. Clearly $y_{1} \notin I$ as $V_{2} \cap I=\phi$. Now $\left(x_{1}-x_{2}\right) y_{1} \in I$ with $x_{1}-x_{2} \notin I$ and $y_{1} \notin I$. Thus $x_{1}-x_{2} \in A$ and $y_{1} \in B$. Hence $x_{1}-x_{2} \in V_{1} \subseteq P_{1}$. Thus $P_{1}$ is an additive subgroup of $N$.

Now let us show that $P_{1}$ is a normal subgroup of $N$. Let $x \in P_{1}$ and $n \in N$. If $x \in I$, then $n+x-n \in I \subseteq P_{1}$. Let us assume $x \in V_{1}$. Then there exists $y \in V_{2}$ such that $x y \in I$. If $n+x-n \in I$, we are done. Let us assume $n+x-n \notin I$. Now $(n+x-n) y=n y-x y-n y \in I$. Thus $n+x-n \in A$ and hence $n+x-n \in V_{1} \subseteq P_{1}$. So $P_{1}$ is a normal subgroup of $N$.

Now we claim that $P_{1}$ is a right ideal of $N$. Let $x \in P_{1}$ and $n \in N$. If $x \in I$, then $x n \in I \subseteq P_{1}$. If $n x \in I$, we are done. So $n x \notin I$ and $x \in V_{1}$. Then there exists $y \in V_{2}$ such that $x y \in I$. Now $(n x) y \in I$ with $n x \notin I$ and $y \notin I$. Thus $n x \in A$ and hence $n x \in V_{1} \subseteq P_{1}$. So $P_{1}$ is a right ideal of $N$.

Now let us show that $P_{1}$ is a left ideal of $N$. Let $x \in P_{1}$ and $n, n^{\prime} \in N$. If $x \in I$, then $n\left(n^{\prime}+x\right)-n n^{\prime} \in I \subseteq P_{1}$. Let us assume that $x \in V_{1}$. If $n\left(n^{\prime}+x\right)-n n^{\prime} \in I$, then we are done. Hence let us assume $n\left(n^{\prime}+x\right)-n n^{\prime} \notin I$. Since $x \in V_{1}$, there exists $y \in V_{2}$ such that $x y \in I$. Clearly $y \notin I$. Now $\left(n\left(n^{\prime}+x\right)-n n^{\prime}\right) y=n\left(n^{\prime} y+x y\right)-n\left(n^{\prime} y\right) \in I$ as $x y \in I$. Thus $n\left(n^{\prime}+x\right)-n n^{\prime} \in$ $V_{1} \subseteq P_{1}$ and hence $P_{1}$ is an ideal of $N$. So $P_{1}$ is an ideal of $N$. Similarly $P_{2}$ is an ideal of $N$.

We now show that $P_{1}$ is a prime ideal of $N$. Let $J$ and $K$ be ideals of $N$ such that $J K \subseteq P_{1}$ and suppose that $J \nsubseteq P_{1}$. Let $j \in J$ but $j \notin P_{1}$. Let $k \in K$. If $k \in I$, then $k \in P_{1}$. Let us assume that $k \notin I$. Clearly $j k \in P_{1}$. If $j k \in I$, then $j \in B$ and $k \in A$ since $j \notin V_{1}$, and hence $k \in V_{1} \subseteq P_{1}$. If $j k \notin I$, then $j k \in V_{1}$ and there exists $y \in V_{2}$ such that $j k y \in I$. Since $P_{2}$ is an ideal, we have $j y \in V_{2}$, and so $k \in V_{1} \subseteq P_{1}$. Thus $P_{1}$ is a prime ideal of $N$. Similarly $P_{2}$ is a prime ideal of $N$.

Note that in Theorem 2.12, the converse is not true in general, as the following example shows. 
Example 2.13. In $N=\mathbb{Z}_{6},\{0\}$ is a completely reflexive ideal and completely semiprime ideal and $\mathbb{Z}_{6}$ has only two prime ideals, but its generalized idealbased zero-divisor graph $\widehat{\Gamma(N)}$ is not a bipartite.

We now also show by an example that the Theorem 2.12 will fail if $I$ is not completely semiprime.

Example 2.14. Let $(N,+)$ (where $N=\{0, a, b, c\}$ ) be the Klein's four group. Define multiplication in $N$ as follows:

\begin{tabular}{c|cccc}
$\cdot$ & 0 & $a$ & $b$ & $c$ \\
\hline 0 & 0 & 0 & 0 & 0 \\
$a$ & 0 & 0 & 0 & 0 \\
$b$ & 0 & 0 & 0 & $a$ \\
$c$ & 0 & 0 & 0 & $a$
\end{tabular}

Then $(N,+, \cdot)$ is a near-ring (see Pilz [10], P-408, Scheme-14). If $I=\{0, a\}$, then $I$ is completely reflexive, but not completely semiprime. Here $\widehat{\Gamma_{I}(N)}$ is a complete bipartite graph but $I$ cannot be written as the intersection of two prime ideals.

Acknowledgment. The authors express their sincere thanks to the referee for his/her valuable comments and suggestions which improve the paper a lot.

\section{References}

[1] D. D. Anderson and M. Naseer, Beck's coloring of a commutative ring, J. Algebra 159 (1993), 500-514.

[2] D. F. Anderson and P. S. Livingston, The zero-divisor graph of a commutative ring, J. Algebra 217 (1999), 434-447.

[3] I. Beck, Coloring of commutative rings, J. Algebra 116 (1988), 208-226.

[4] M. Behboodi and R. Beyranvand, Strong zero-divisor graphs of non-commutative rings, International Journal of Algebra 2 (2007), no. 1, 25-44.

[5] J. A. Bondy and U. S. R. Murty, Graph Theory with Applications, North-Holland, Amsterdam, 1976.

[6] A. Cannon, K. Neuerburg, and S. P. Redmond, Zero-divisor graph of anear-rings and semigroups, in: H. Kiechle, A. Kreuzer, M.J.Thomsen (Eds), Nearrings and Nearfields, Springer, Dordrecht, The Netherlands, 2005, 189-200.

[7] F. R. DeMeyer, T. McKenzie, and K. Schneider, The zero-divisor graph of a commutative semigroups, Semigroup Forum 65 (2002), 206-214.

[8] P. Dheena and B. Elavarasan, An ideal based-zero-divisor graph of 2-primal near-rings, (Submitted).

[9] S. B. Mulay, Cycles and symmetries of zero-divisors, Comm. Algebra 30 (2002), no. 7, 3533-3558.

[10] G. Pilz, Near-Rings, North-Holland, Amsterdam, 1983.

[11] S. P. Redmond, An ideal-based zero-divisor graph of a commutative ring, Comm. Algebra 31 (2003), no. 9, 4425-4443. 
Patchirajulu Dheena

Department of Mathematics

ANNAMALAi UNIVERSiTy

ANNAMALAINAGAR - 608002, INDIA

E-mail address: dheenap@yahoo.com

BALASUBRAMANiAn Elavarasan

Department of Mathematics

K. S. R. College of Engineering

Tiruchengode - 637 215, Namakkal Dt, TAmilnadu, India

E-mail address: belavarasan@gmail.com 